\title{
Minimal Cytological Changes of Progressive Supranuclear Palsy Type in Clinically Possible Progressive Supranuclear Palsy
}

\author{
S. Toru ${ }^{\mathrm{a}}$ T. Uchihara ${ }^{\mathrm{b}}$ T. Akashic T. Kobayashi ${ }^{\mathrm{a}}$ \\ ${ }^{a}$ Department of Neurology, Nakano General Hospital, bepartment of Neurology, Tokyo Metropolitan Institute for \\ Neuroscience, and ${ }^{\mathrm{C}}$ Department of Pathology, Tokyo Medical and Dental University, Tokyo, Japan
}

Dear Sir,

Progressive supranuclear palsy (PSP), first described in 1964 [1], is a part of the atypical parkinsonian syndrome. Here we report a patient with the clinical diagnosis of possible PSP, based on the proposed clinical criteria [2]. The brain contained microscopic lesions positive for 4-repeat (4R) tau compatible with PSP, but the frequency of lesions was much less than that defined by current operational criteria for histological diagnosis of PSP $[3,4]$.

\section{Case Report}

A 69-year-old man experienced gradual onset of gait disturbance. He had undergone surgery for spinal canal stenosis, cancer in the ascending colon, and groin hernia. He had been working as a dentist, had not smoked and drank little alcohol. His family history was negative for similar neurological deficits, and no consanguinity was reported.

He became aware of his gait disturbance at age 67. Its rapid progression prompted him to consult our neurology department. Retropulsion, brachybasia and wide-based gait were noted. He had no tremor at rest. L-DOPA (up to $300 \mathrm{mg}$ daily), amantadine hydrochloride, Lthreo-DOPS, and tandospirone citrate were not effective. He was hospitalized 2 months later because of fever up to $39.2^{\circ} \mathrm{C}$. On neurological examination, he was fully conscious but apathic and had little vigor. He also had impaired concreteness in thinking, slowed information processing and decreased verbal fluency. He had apparent dementia and scored 14/30 points on HDS-R (Hasegawa Dementia Rating Scale - Revised) showing disturbance in orientation, recent memory, and word remembrance and recall. Extraocular movements were normal, except for some slowing of vertical saccades. His face was oily and mask-like. Myerson sign and palmomental reflex were absent. Rigidity was marked in his neck and to a lesser extent in his arms. Bradykinesia, dysarthria and dysphagia were also present. Postural tremor was observed in the limbs and the trunk. Limb ataxia was seen in his left arm. Neither motor weakness nor sensory disturbance was found. It was impossible for him to maintain a sitting position or stand up. Deep tendon reflexes were diminished in the distal part of extremities. Babinski and Chaddock signs were flexor.

Laboratory examinations disclosed slight anemia and hypo-albuminemia. Chronic atrial fibrillation was noted. A chest roentgenogram was normal. Diffuse brain atrophy was seen on brain MRI, but there was no obvious atrophy of the tec- tum mesencephali (fig. 1a). MRI also showed an old infarction in the left cerebellar hemisphere but ischemic changes in the deep white matter remained mild.

The clinical diagnosis was compatible with 'possible PSP' according to NINDSSPSP criteria [2] based on his gradually progressive, adult-onset neurological disease with vertical saccades slowing and prominent postural instability with falls within the year after disease onset. Proximal dominant rigidity, L-DOPA unresponsiveness, dysarthria, dysphagia, and cognitive impairment from the early stage supported this diagnosis.

Repeated episodes of aspiration pneumonia were mitigated by antibiotics, but he died of sudden cardiopulmonary arrest 5 months after admission. His entire clinical course lasted 2 years and 5 months.

Brain weight was $1,510 \mathrm{~g}$. Macroscopically, the anterior horns of the lateral ventricles were slightly enlarged, suggesting questionable atrophy of the frontal lobes. An ischemic focus was noted in the left cerebellar hemisphere. Neither discoloration nor atrophy was detected in the basal ganglia, substantia nigra (fig. 1b) or locus ceruleus. The cerebellar dentate nucleus and tegmentum of the pons were normal. Nigral neurons were well preserved without gliosis or extraneuronal free melanin (fig. 1c). A few tau(AT8)/Gallyas-pos-

\section{KARGER}

Fax +4161306 1234 E-Mail karger@karger.ch www.karger.com
Dr. Shuta Toru

Department of Neurology, Nakano General Hospital

4-59-16 Chuo, Nakano-ku

Tokyo 164-8607 (Japan)

Tel. +81 33382 1231, Fax +81 33382 1588, E-Mail shuta-toru@ nakanosogo.or.jp 
Table 1. Distribution of tau/Gallyas-positive lesions between typical and atypical PSP and this case

\begin{tabular}{|c|c|c|c|c|}
\hline \multirow[t]{2}{*}{ Brain area } & \multirow{2}{*}{$\begin{array}{l}\text { Typical } \\
\text { PSP }\end{array}$} & \multirow{2}{*}{$\begin{array}{l}\text { Atypical } \\
\text { PSP }\end{array}$} & \multicolumn{2}{|l|}{ This case } \\
\hline & & & neuronal & glial \\
\hline Globus pallidus & +++ & ++ & absent & rare \\
\hline Subthalamic nucleus & +++ & ++ & rare & absent \\
\hline Substantia nigra & +++ & + & rare & absent \\
\hline Pons & ++ & + & absent & absent \\
\hline Striatum & ++ & + & absent & rare \\
\hline Oculomotor & ++ & + & not included & \\
\hline Medulla & ++ & + & absent & absent \\
\hline Dentate & ++ & + & absent & absent \\
\hline Locus ceruleus & & & + & absent \\
\hline Cortex & + & $+/-$ & rare & rare \\
\hline Central gray & & & ++ & absent \\
\hline
\end{tabular}

At $\times 250$ magnification: $-=$ absent or rare; $+=$ low density (1 or 2 neurons with neurofibrillary tangles (NFTs) or neuropil threads); $++=$ high density (2-5 neurons with NFTs or neuropil threads); $+++=$ very high density (6 or more neurons with NFTs or neuropil threads). Hauw et al. [3] found NFTs and neuropil threads in the structures in bold.

Fig. 1. Brain MRI and neuropathological findings. a Midsagittal T1-weighted image of the brain showing no atrophy of the tectum mesencephali. b Well-pigmented substantia nigra. c Well-preserved nigral neurons without extracellular melanin nor gliosis HE. Scale bar $=100 \mu \mathrm{m}$. d Intranuclear inclusions (Marinesco bodies) in nigral neurons. Ubiquitin immunostain. Scale bar $=20$ $\mu \mathrm{m}$. e Well-preserved neurons in subthalamic nucleus. HE. Scale bar $=50 \mu \mathrm{m}$. f A few neurofibrillary tangles in the subthalamic nucleus. Bodian stain. Scale bar $=10 \mu \mathrm{m}$. g Tuft-shaped astrocytes (asterisk) in the premotor area of frontal cortex. Gallyas silver stain. Scale bar $=50 \mu \mathrm{m}$. h Pretangle neuron and tuft-shaped astrocyte (asterisk) positive for 4-repeat tau-specific antibody. RD4 immunostain, premotor area. Scale bar $=25 \mu \mathrm{m}$.
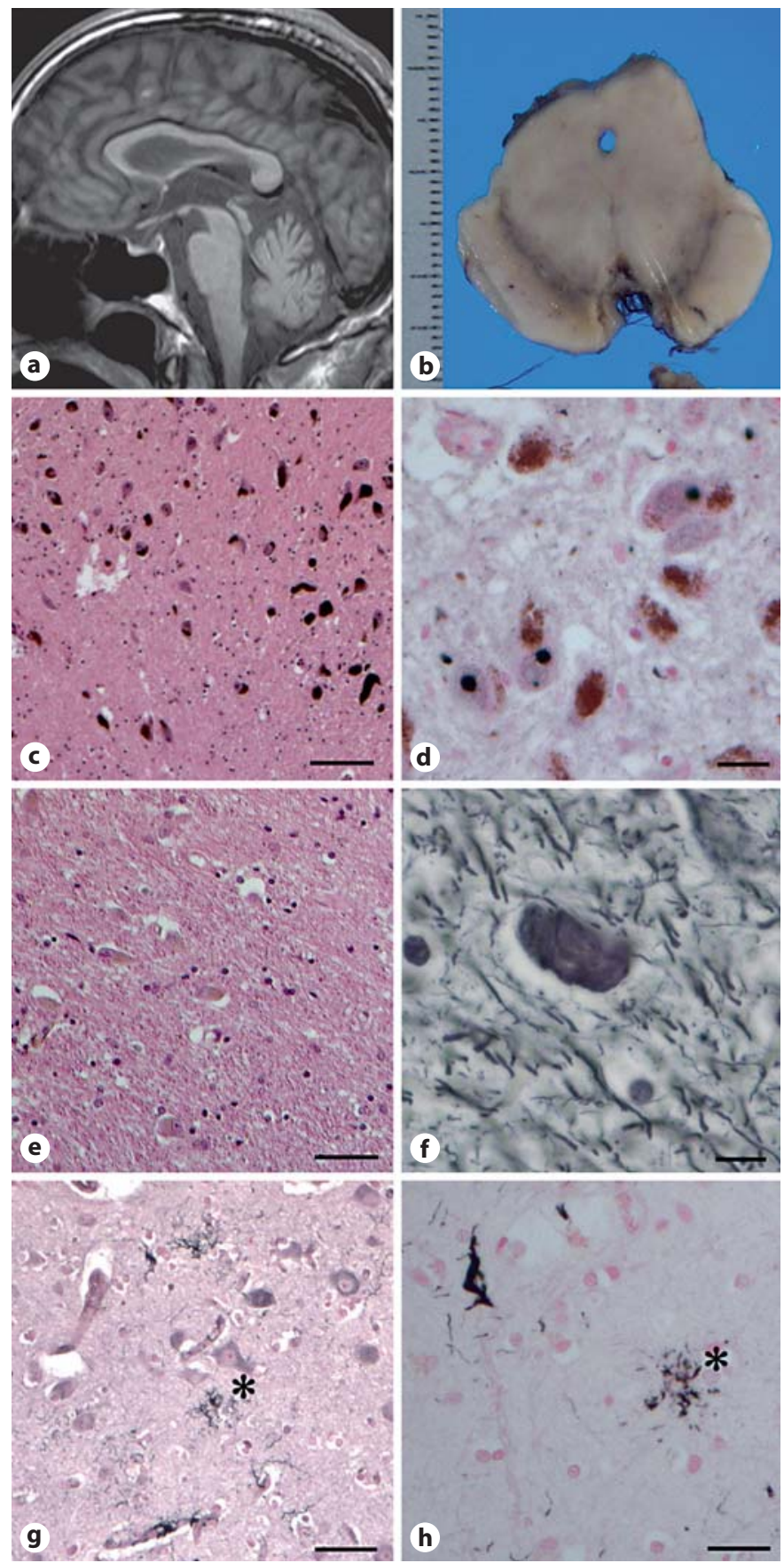

itive neurons and frequent neuronal intranuclear inclusions positive for ubiquitin (Marinesco bodies) were present in the substantia nigra. Neurons and myelin in the subthalamic nucleus were preserved as well (fig. 1e) without gliosis examined on GFAP immunostaining. Neurofibrillary tangles were extremely rare in the subthalamic nucleus (fig. $1 \mathrm{f}$ shows 1 or 2 neurofi- brillary tangles in the entire section). In the premotor area of the frontal cortex, a few Gallyas-positive glia (fig. 1g; tuftshaped astrocytes and coiled bodies) and neurons were immunoreactive for $4 \mathrm{R}$ tauspecific antibody $[1: 1,000, \mathrm{RD} 4$ (Upstate Inc., Charlesville, Va., USA), pretreated with formic acid followed by autoclaving [5]; fig. 1h] but not for 3-repeat tau-specif- ic counterpart (RD3, 1:3,000; Upstate; data not shown). A few glial cells in the globus pallidus exhibited similar staining profiles (data not shown). Semiquantitative assessment of tau-positive lesions are listed in table 1. Immunohistochemisitry with anti-phosophorylated $\alpha$-synuclein (psyn No. 64, 1:10,000; Wako, Tokyo, Japan) demonstrated Lewy bodies to be restricted 
to the dorsal vagal nucleus and locus ceruleus. Some neurofibrillary tangles were present in the central gray, entorhinaltransentorhinal cortex, but only a few in the subiculum - CA1. Argyrophilic grains were absent in the hippocampus and amygdaloid nuclei (AT8 immunostain and Gallyas silver stain). Cytoplasmic staining for phosphorylated-TDP43 was absent.

\section{Discussion}

PSP was first described as a distinct entity in 1964 by Steele et al. [1]. Clinical and pathological diagnostic criteria, proposed between 1994 and 1996 by the NINDS-III [2-4] are in current use. The clinical picture of this patient was compatible with 'possible PSP' [2] or 'PSP' [6] according to these criteria. The small number of lesions identified at autopsy, however, were far below the threshold of the current quantitative histopathological criteria for PSP, as shown in table 1 . Is it, then, reasonable to conclude that the final diagnosis is not PSP? In this patient, the presence of cytological changes (4R tau-positive coiled bodies, astrocytes and neurons) in the premotor cortex is compatible with cytopathological diagnosis of PSP, but their quantities were far below the required thresholds.

Because the clinical course of this patient was relatively short, these slight cytopathological changes may possibly represent a very early stage of PSP. Although the mean survival of patients with PSP after its onset is estimated to be 5.6 years [7], this patient died suddenly of suffocation approximately 2.5 years after the onset. One explanation for the scarcity of lesions is that the disease process might have been truncated in its early stage. In a report of 2 early PSP cases, neurofibrillary tangles were limited to the locus ceruleus, third cranial nerve complex, nucleus supratrochlearis, nucleus centralis superior, and nucleus basalis of Meynert [8]. However, those cases did not satisfy the clinical criteria even for possible PSP because gaze palsy was absent. Recently, another report showed early PSP-like lesions in the striatum, followed by the globus pallidus/subthalamus and selected nuclei of the brain stem in cases with neurologically normal or mild cognitive impairment alone [9].Interestingly, it has been reported that the pathological tau burden is negatively correlated with the disease duration, indicating that a larger quantity of tau lesions and their extension are related to fulminant disease with a shorter clinical course leading to early death [10]. Whether an abundant tau burden in PSP cases of short duration can be extrapolated to our patient who had premature truncation of the disease as well has yet to be clarified.

One may wonder whether this patient had a disease other than PSP. The pathological examination, however, failed to identify any of the specific features of diseases that may cause parkinsonism (postencephalitic parkinsonism, vascular parkinsonism, multiple system atrophy, corticobasal degeneration) and dementias (Alzheimer's disease, Pick body disease and vascular dementia) except for Lewy bodies in the locus ceruleus and dorsal motor nucleus of vagus. Because neither neuronal loss nor Lewy bodies were present in the substantia nigra, it is questionable whether the mere presence of Lewy bodies in these nuclei adequately explains clinical features of this patient, such as levodopa-unresponsive parkinsonism with gait and postural abnormalities. A recent study confirmed that incidental presence of Lewy bodies in the brainstem in individuals without parkinsonism (incidental Lewy body disease) is not rare [11]. However, it is still possible that the mild PSP cytopathology and small number of extranigral Lewy bodies of this patient have synergistic effects to generate the clinical picture of PSP. As candidate states possibly related to PSP, pure akinesia and pallidonigroluysian atrophy should be considered. The clinical findings of this patient are not compatible with pure akinesia, a clinical concept [12], because of the early presence of rigidity and cognitive impairment. The absence of neuronal loss in the globus pallidus and substantia nigra [13] is not compatible with histopathological diagnosis of pallidonigroluysian atrophy. Although a recent study claimed a possible relevance of nigral Marinesco bodies in the development of extrapyramidal features [14], it remains to be clarified whether numerous Marinesco bodies without neuronal loss lead to extrapyramidal manifestations. Furthermore, it is doubtful that $\mathrm{Ma}-$ rinesco bodies are related to more extended clinical pictures compatible with PSP. We were left with the PSP-type cytopathology as the only substrate that may explain the PSP-type clinical features of this patient. Otherwise, this patient's PSP-like clinical features and cytopathology would be only by chance without mutual relevance, which is unlikely. Because the pathological diagnostic criteria proposed by the NINDS-III were designed to isolate authentic PSP by excluding atypical PSP, they do not tell us how the amount of lesions is related to the clinical picture. Despite the existing clinical and pathological criteria for PSP, it is not clear to what extent the clinical and the histological features are related in an individual patient. Because the cytopathological changes reported here usually are not seen in normal aging, their presence, even in small amounts, strongly suggests a pathological process specific to PSP. This patient with the clinical picture of PSP but a limited amount of PSP-type cytopathology without overt neuronal loss provides a unique opportunity to reconsider cytopathological changes of PSP type and their relationship to neuronal loss and clinical manifestations, and finally therapeutic intervention.

\section{References}

1 Steele JC, Richardson JC, Olszewski J: Progressive supranuclear palsy. Arch Neurol 1964;10:333-359.

2 Litvan I, Agid Y, Calne D, et al: Clinical research criteria for the diagnosis of progressive supranuclear palsy (Steele-RichardsonOlszewski syndrome): report of the NINDS-SPSP international workshop. Neurology 1996;47:1-9.

3 Hauw JJ, Daniel SE, Dickson D, et al: Preliminary NINDS neuropathologic criteria for Steele-Richardson-Olszewski syndrome (progressive supranuclear palsy). Neurology 1994;44:2015-2019.

4 Litvan I, Hauw JJ, Bartko JJ, et al: Validity and reliability of the preliminary NINDS neuropathologic criteria for progressive supranuclear palsy and related disorders. Neuropathol Exp Neurol 1996;55:97-105.
5 de Silva, Lashley T, Gibb G, et al: Pathological inclusion bodies in tauopathies contain distinct complements of tau with three or four microtubule-binding repeat domains as demonstrated by new specific monoclonal antibodies. Neuropathol Appl Neurobiol 2003;29:288-302. 
6 Collins SJ, Ahlskog JE, Parisi JE, et al: Progressive supranuclear palsy: neuropathologically based diagnostic clinical criteria. J Neurol Neurosurg Psychiatry 1995;58:167173.

7 Litvan I, Mangone CA, McKee A, et al: Natural history of progressive supranuclear palsy (Steele-Richardson-Olszewski syndrome) and clinical predictors of survival: a clinicopathological study. J Neurol Neurosurg Psychiatry 1996;60:615-620.

8 Kleinschmidt-DeMasters BK: Early progressive supranuclear palsy: pathology and clinical presentation. Clin Neuropathol 1989;8: 79-84.
Santpere G, Ferrer I: Delineation of early changes in cases with progressive supranuclear palsy-like pathology: astrocytes in striatum are primary targets of tau phosphorylation and GFAP oxidation. Brain Pathol 2009;19:177-187.

10 Williams DR, Holton JL, Strand C, et al: Pathological tau burden and distribution distinguishes progressive supranuclear palsy-parkinsonism from Richardson's syndrome. Brain 2007;130:1566-1576.

11 Beach TG, Adler CH, et al: Unified staging system for Lewy body disorders. Acta Neuropathol 2009:117:613-634.
12 Narabayashi H, Imai $H$, Yokochi $M$, et al: Cases of pure akinesia without rigidity and tremor with no effect by L-Dopa therapy; in Birkmayer W, Horniekiewicz O (eds): Advances in Parkinsonism. Basel, Roche, 1976, pp 335-342.

13 Jellinger K: Pallidal, pallidonigral and pallidoluysionigal degenerations including association with thalamic and dentate degeneration; in Vinken PJ et al. (eds): Handbook of Clinical Neurology, Amsterdam, Elsevier, 1986, vol 5, p 445.

14 Beach TG, Walker DG, Sue LI, et al: Substantia nigra Marinesco bodies are associated with decreased striatal expression of dopaminergic markers. J Neuropathol Exp Neurol 2004:63:329-337. 